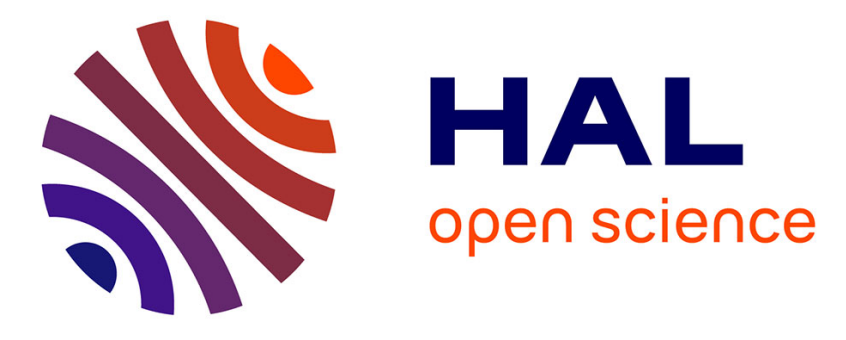

\title{
Relation between interlayer exchange coupling and nonferromagnetic behavior of $\mathrm{Fe}$ in $\mathrm{Ni} / \mathrm{Fe} / \mathrm{Co}$ superlattices
}

\author{
A. Ziane, F. Amitouche, A. Hadj-Larbi, S. Bouarab, C. Demangeat
}

\section{To cite this version:}

A. Ziane, F. Amitouche, A. Hadj-Larbi, S. Bouarab, C. Demangeat. Relation between interlayer exchange coupling and nonferromagnetic behavior of $\mathrm{Fe}$ in $\mathrm{Ni} / \mathrm{Fe} / \mathrm{Co}$ superlattices. Physical Review B: Condensed Matter and Materials Physics (1998-2015), 2006, 73, pp.064411. 10.1103/PhysRevB.73.064411 . hal-00211562

\section{HAL Id: hal-00211562 \\ https://hal.science/hal-00211562}

Submitted on 21 Feb 2020

HAL is a multi-disciplinary open access archive for the deposit and dissemination of scientific research documents, whether they are published or not. The documents may come from teaching and research institutions in France or abroad, or from public or private research centers.
L'archive ouverte pluridisciplinaire HAL, est destinée au dépôt et à la diffusion de documents scientifiques de niveau recherche, publiés ou non, émanant des établissements d'enseignement et de recherche français ou étrangers, des laboratoires publics ou privés. 


\title{
Relation between interlayer exchange coupling and nonferromagnetic behavior of Fe in $\mathrm{Ni} / \mathrm{Fe} / \mathrm{Co}$ superlattices
}

\author{
A. Ziane, F. Amitouche, A. Hadj-Larbi, and S. Bouarab \\ Laboratoire de Physique et Chimie Quantique, Université Mouloud Mammeri de Tizi-Ouzou, 15000 Tizi-Ouzou, Algeria \\ C. Demangeat \\ Institut de Physique et de Chimie des Matériaux de Strasbourg, 23, Rue du Loess, B.P. 43, F-67034 Strasbourg Cedex 2, France \\ (Received 15 May 2005; revised manuscript received 9 November 2005; published 9 February 2006)
}

\begin{abstract}
Recent experimental studies, using a photoemission electron microscope on a $\mathrm{Ni} / \mathrm{Fe} / \mathrm{Co} / \mathrm{Cu}(100)$ system, found that the $\mathrm{Fe}$ layers at both the $\mathrm{Fe} / \mathrm{Co}$ and $\mathrm{Fe} / \mathrm{Ni}$ interfaces are ferromagnetically ordered, and that there is an oscillatory intercoupling between the Co and Ni layers across the Fe spacer. In the present paper we performed self-consistent $a b$ initio calculations to determine the magnetic profile and the interlayer exchange coupling (IEC) in fcc $\mathrm{Ni} / \mathrm{Fe}_{n} / \mathrm{Co}(100)$ superlattices, for different thicknesses of the Fe spacer $(n=1-7)$. The compositional ordering at the $\mathrm{Fe} / \mathrm{Co}$ and $\mathrm{Fe} / \mathrm{Ni}$ interfaces leads to a completely different IEC behavior. Strong $\mathrm{Fe}-\mathrm{Ni}(\mathrm{Co})$ ferromagnetic coupling with enhanced $\mathrm{Fe}$ magnetic moment are always obtained in the intermixed layers.
\end{abstract}

DOI: 10.1103/PhysRevB.73.064411

\section{INTRODUCTION}

Magnetic multilayers are of increasing interest for their new and exciting coupling phenomena and related effects such as oscillatory exchange coupling, giant magnetoresistance (GMR) and tunneling magnetoresistance (TMR). This has opened a very large application field in the scope of the magnetic recording such as sensitive read heads. Besides, a new class of materials of metastable structures with novel and unusual magnetic properties which can be stabilized by epitaxial growth, have furthermore augmented the interest in investigating the magnetic multilayers. In this context the $\mathrm{Fe} / \mathrm{Ni}$ (Refs. 1-6) and Fe/Co (Refs. 7 and 8) multilayers of face centered cubic (fcc) stacking show interesting structural and magnetic properties even at room temperature. In the present case the $\mathrm{Fe}$ spacer is very different from its usual bcc ferromagnetic (FM) ground state. It presents mainly a nonferromagnetic behavior with sizable magnetic moments, higher not only than those of $\mathrm{Ni}$ but also than those of Co atoms.

Recently there have been much concern about the description of the magnetism of the fcc iron. Fcc-Fe is a high temperature phase and does not present usually a long range magnetic order like the ferromagnetic ordering in bcc-Fe. However a small modification of its lattice parameter can induce an onset of various kind of long range magnetism order whose determination is still a matter of debate. ${ }^{9-11}$ Moreover, this nonferromagnetic bulk fcc-Fe is like most of the 3- $d$ transition metal elements very suitable to support ferromagnetism when it is in contact with a strong ferromagnet like $\mathrm{Ni}$ or $\mathrm{Co}$.

By using X-ray magnetic circular dicroism (XMCD), Dallmeyer et $\mathrm{al}^{8}{ }^{8}$ have shown that the fcc-Fe films mediate an oscillatory indirect coupling in $\mathrm{Co} / \mathrm{Fe} / \mathrm{Co}(100)$ structures that alternates in correspondence with the changes of the $\mathrm{Fe}$ magnetization. Escorcia-Aparitio et al. ${ }^{7}$ explored the $\mathrm{Co} / \mathrm{Fe} / \mathrm{Co} / \mathrm{Cu}(100)$ system with a magneto-optic Kerr effect (MOKE) technique. Their results show an antiferromagnetic
PACS number(s): 75.70.Cn, 75.70.Kw, 78.20.Ls

(AF) interlayer coupling between Co layers around 7 and 18 $\mathrm{Fe}$ monolayers (ML's) thicknesses. Recently, Kenta et al. ${ }^{12}$ investigated $\mathrm{Fe} / \mathrm{Ni} / \mathrm{Cu}(100)$ films by a depth-resolved $\mathrm{XMCD}$ technique and found an oscillatory behavior in the Fe magnetization as a function of $\mathrm{Fe}$ thickness.

All experiments show that Fe films should undergo some magnetic interactions with $\mathrm{Ni}(\mathrm{Co})$ substrates, but the situation was not enough explored when Fe is inserted between $\mathrm{Ni}(100)$ and $\mathrm{Co}(100)$ slabs. In this context a few group of experimentalists have recently devoted their work to the determination of the magnetic polarization and interlayer exchange coupling (IEC) of Ni/Fe/Co superlattices. ${ }^{13-16} \mathrm{Gen}-$ erally they first grow $\mathrm{Co}$ on a $\mathrm{Cu}$ seed layer and so, $\mathrm{Fe}$ and $\mathrm{Ni}$ films are then grown into crossed wedges to vary their thickness independently. The use of $\mathrm{Ni}$ instead of $\mathrm{Co}$ as the top magnetic layer has the advantage that the magnetic signal of each layer can be determined separately, allowing the layer resolved characterization of the entire system. ${ }^{13}$ Various experimental techniques have been used [XMCD, photoemission electron microscope (PEEM),...] and a consistent model has been deduced from those experiments:

(i) Fe presents a long range polarization which is essentially of the nonferromagnetic type contrary to bcc-Fe which is ferromagnetic;

(ii) at the interface with $\mathrm{Co}(\mathrm{Ni})$ the magnetism of $\mathrm{Fe}$ is typically parallel to that of $\mathrm{Co}(\mathrm{Ni})$;

(iii) an interlayer exchange coupling between $\mathrm{Co}$ and $\mathrm{Ni}$ through the fcc-Fe spacer is present, but its period is not well defined.

From the theoretical point of view several investigations on the magnetism of the interface layers were performed. More precisely, band structure calculations suggest that in fcc Fe system there exist a variety of magnetic phases with the spacer thickness. However, density functional calculations concerning the electronic and magnetic structures of $\mathrm{Ni} / \mathrm{Fe} / \mathrm{Co}$ superlattices have not appeared yet. We can however notice the works on $\mathrm{Fe}$ thin films on Co substrate by Mokrani et al. ${ }^{17}$ and by Spisák and Hafner. ${ }^{18}$ Mokrani et al. 
have determined the magnetic map of 1-4 Fe ML's on Co using the tight-binding linear muffin tin orbital (TB-LMTO) method within the local density approximation (LDA). They have found that the interfacial $\mathrm{Fe} / \mathrm{Co}$ coupling is always of ferromagnetic-type, and that the ferromagnetic solution is the ground state only up to three Fe ML's. However it is well known that LDA leads to a wrong ground state of $\mathrm{Fe}$ as discussed by Wang et al. ${ }^{19}$ Therefore, as pointed out by Spisák and Hafner, the generalized gradient approximation (GGA) should be used. Those authors have investigated the $\gamma$-Fe ultrathin films on fcc $\mathrm{Co}(100)$ substrates with $a b$ initio local spin density method including GGA, for different $\mathrm{Fe}$ thicknesses and for relaxed and unrelaxed interlayer distances. They found that the interfacial $\mathrm{Fe} / \mathrm{Co}$ and $\mathrm{Fe}$ (surface)/Fe(subsurface) coupling is always ferromagnetic. They found also that the intermixing at the $\mathrm{Fe} / \mathrm{Co}$ interface stabilizes the three Fe ML's with FM configuration and that for 4 Fe ML's the FM phase is also preferred. Indeed, the magnetic properties of the multilayers depend strongly on their microstructure and the quality of the interfaces. ${ }^{20-22}$ Later on, Hadj-larbi et al ${ }^{23}$ have considered the system $\mathrm{Ni} / \mathrm{Fe} / \mathrm{Ni}(100)$ by considering both abrupt $\mathrm{Fe} / \mathrm{Ni}$ interfaces and some ordered alloys at the interface following the approach proposed by Freyss et al. ${ }^{24}$ for Fe/Cr superlattices. Because $\mathrm{Fe}-\mathrm{Ni}$ alloys exist in nature, experimentalists cannot avoid some kind of interdiffusion in the preparation of the superlattices. Consequently, for $\mathrm{Ni} / \mathrm{Fe} / \mathrm{Ni}(100)$ superlattices, ${ }^{23}$ mixed interfaces have lower energy as compared to abrupt interfaces. Moreover, the description of the magnetism of $\mathrm{Fe}$ in contact with $\mathrm{Ni}$ appears to be a timely subject. For example, Mavropoulos et al. ${ }^{25}$ have derived within the Korringa, Kohn, and Rostoker (KKR) calculation the spin moments of Fe clusters, with different sizes, on $\mathrm{Ni}(001)$ surfaces. They found that the average spin moments of Fe decreases with the cluster size.

In this work we present an ab initio study of the magnetic properties of the $\mathrm{Ni}_{5} / \mathrm{Fe}_{n} / \mathrm{Co}_{5}$ superlattices for $n=1-7$. The interesting point of these systems is the competition between the $\mathrm{Fe}-\mathrm{Ni}$ and $\mathrm{Fe}-\mathrm{Co}$ ferromagnetic couplings at the interface and the antiferromagnetic order that could occur in the iron spacer. We have used the TB-LMTO method in the atomic sphere approximation (ASA), in order to discuss the magnetic polarization of the $\mathrm{Fe}$ spacer in $\mathrm{Ni} / \mathrm{Fe} / \mathrm{Co}$ superlattices and the resulting IEC for both abrupt and mixed interfaces. In Sec. II we are reminded briefly of the model used for the calculations. Section III is devoted to the determination of the magnetic map of $\mathrm{Ni}_{5} / \mathrm{Fe}_{n} / \mathrm{Co}_{5}$ superlattices ( $n$ $=1, \ldots, 7)$. By considering the difference between the total energies with $\mathrm{Ni}$ and Co blocks aligned, ferromagnetically and antiferromagnetically, we deduce the IEC. In Sec. IV we perform similar calculations with ordered alloys at the $\mathrm{Fe} / \mathrm{Co}$ and $\mathrm{Fe} / \mathrm{Ni}$ interfaces. This induces great modifications of the IEC. Section V is devoted to the conclusion.

\section{CALCULATION MODEL}

As mentioned in the Introduction, the calculations are performed using scalar-relativistic version of the $k$-space TBLMTO method ${ }^{26,27}$ with the atomic sphere approximation.
This method consists of resolving self-consistently the KohnSham equations in a minimum basis approach. The choice of the GGA for exchange and correlation functional was discussed in detail in a previous work. ${ }^{23}$ This choice was dictated mainly by the fact that GGA due to Langreth-Mehl-Hu functional yields accurate bulk properties for both fcc Ni and Fe elements. With this functional the lattice parameter $a_{\text {Co }}$ $=6.63$ a.u. of bulk fcc-Co was obtained by the minimization of the total energy. This value leads to bulk magnetic moment of $1.62 \mu_{B}$. Hadj-Larbi et al. ${ }^{23}$ obtained for bulk fcc-Ni a lattice parameter of 6.63 a.u. with a magnetic moment of $0.63 \mu_{B}$, whereas the ground state of bulk fcc-Fe was obtained for $a_{\mathrm{Fe}}=6.56$ a.u. with a magnetic moment of $1.0 \mu_{B}$. However, for $a_{\mathrm{Fe}}=6.63$ a.u. (parameter of Co), the Fe moment is equal to $1.2 \mu_{B}$.

To simulate $\mathrm{Ni} / \mathrm{Fe} / \mathrm{Co}$ multilayers we have used the well known supercell technique consisting of a periodical repetition of a thin symmetry preserving films. We have fixed the thickness of both Ni and Co slabs to 5 ML's and varied the number of Fe ML's from 1 to 7 , in order to study the dependence of the IEC on the $\gamma$-Fe thickness. It is well known that the calculation of the equilibrium $\mathrm{Ni}-\mathrm{Fe}$ and $\mathrm{Co}-\mathrm{Fe}$ distances by means of the ASA is somewhat incorrect essentially because of the anisotropic deformation in the energy changes ${ }^{28}$ during the relaxation. For all superlattices studied in the present paper we have considered epitaxial growth with the same lattice parameter $\left(a_{\mathrm{Co}}\right)$. We have doubled the unit cell in the (100) plane in order to simulate a some kind of intermixing at the interface and also to search for a possible inplane AF arrangement in the Fe spacer layers. The interdiffusion at the $\mathrm{Fe} / \mathrm{Ni}$ and $\mathrm{Fe} / \mathrm{Co}$ interfaces were modeled via monolayers containing both $\mathrm{Fe}$ and $\mathrm{Ni}$ (at the $\mathrm{Ni}$ interface) and $\mathrm{Fe}$ and $\mathrm{Co}$ (at the $\mathrm{Co}$ interface) in a $\mathrm{Fe}_{0.5} \mathrm{Ni}_{0.5}$ $\left(\mathrm{Fe}_{0.5} \mathrm{Co}_{0.5}\right)$ ordered way by considering the superlattices $\mathrm{Ni}_{4} / \mathrm{Fe}_{0.5} \mathrm{Ni}_{0.5} / \mathrm{Fe}_{n-1} / \mathrm{Fe}_{0.5} \mathrm{Co}_{0.5} / \mathrm{Co}_{4}(n=1-7)$. As usual we have determined the IEC by calculating the difference $E_{\mathrm{FM}}$ $-E_{\mathrm{AF}}$ between the total energies obtained for the two opposite interlayer magnetic arrangements $\mathrm{FM}$ and $\mathrm{AF}$. The $\mathrm{FM}(\mathrm{AF})$ arrangement corresponds to parallel (antiparallel) magnetizations of two successive Co and Ni slabs. In the two cases (abrupt and mixed interfaces), the calculations are performed using an increasing number of $k$-points in the irreducible Brillouin zone until convergence which is achieved for $144 k$-points.

\section{MAGNETIC MAP AND IEC OF $\mathrm{Ni}_{5} \mathrm{Fe}_{n} / \mathrm{Co}_{5}(n=1, \ldots, 7)$}

In this part, we focus our attention on the determination of both IEC and the magnetic moments distribution as a function of the Fe spacer thickness $(n=1-7)$ for $\mathrm{FM}$ and $\mathrm{AF}$ arrangements between $\mathrm{Co}$ and $\mathrm{Ni}$ slabs in $\mathrm{Ni}_{5} / \mathrm{Fe}_{n} / \mathrm{Co}_{5}(001)$ superlattices with abrupt interfaces. Input parameters are those usually obtained in the bulk cases whereas the calculations are started from all possible initial spin configurations in the Fe spacer. We obtained many different converged solutions as already discussed by Asada and Blügel ${ }^{29}$ in the case of fcc-Fe on $\mathrm{Cu}(100)$. However, by considering two inequivalent magnetic atoms per layer we went beyond the layered antiferromagnetic configurations described by Asada 


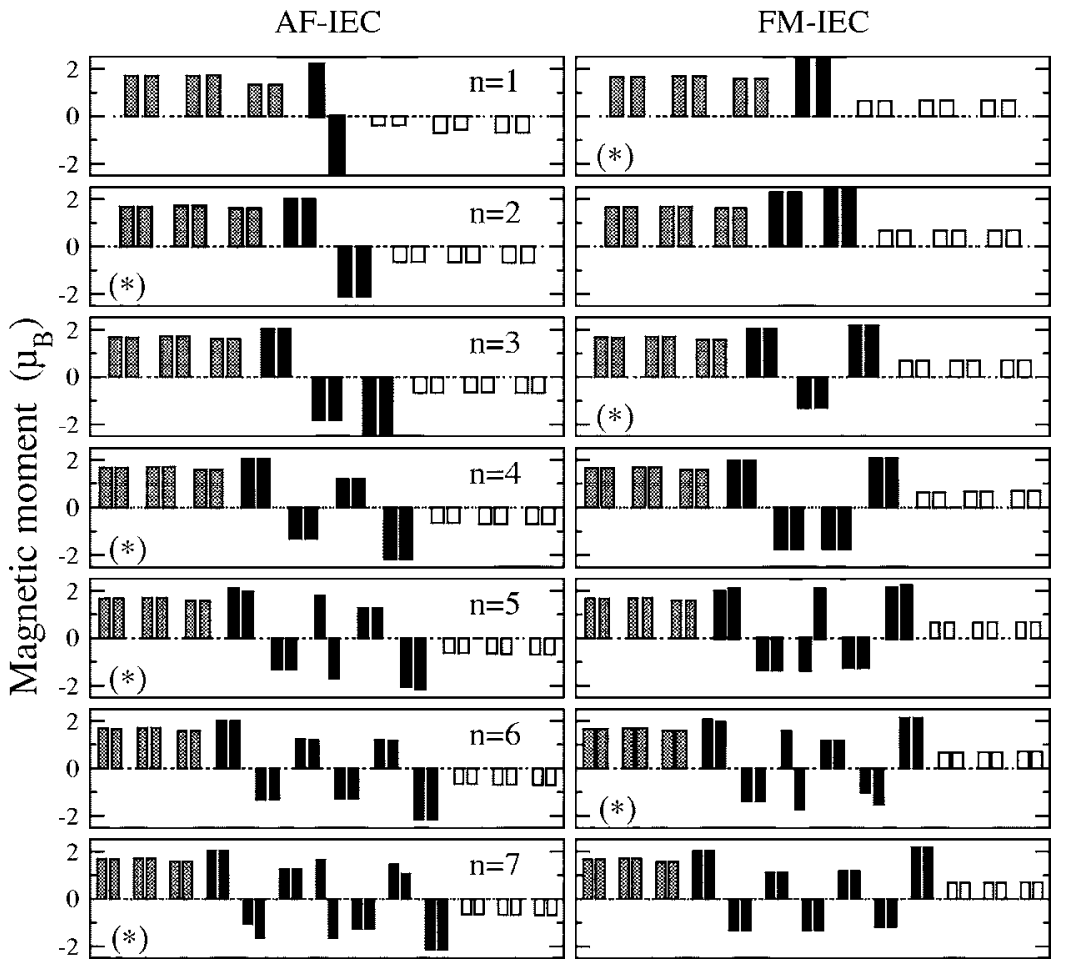

FIG. 1. Magnetic profiles (in $\left.\mu_{B}\right)$ of $\mathrm{Ni}_{5} / \mathrm{Fe}_{n} / \mathrm{Co}_{5}(n=1, \ldots, 7)$ superlattices for AF and FM alignment between $\mathrm{Co}$ and $\mathrm{Ni}$ slabs (left and right panels, respectively). Dark, gray, and open bars represent the values of the magnetic moments of $\mathrm{Fe}, \mathrm{Co}$, and $\mathrm{Ni}$ atoms, respectively. The most stable configuration is indicated by (*). Two magnetically inequivalent atoms per plane have been considered.

and Blügel. In a few cases, an in-plane antiferromagnetic configuration was obtained in one Fe-monolayer located near the middle of the Fe-spacer. In the present paper we are not really interested in repeating the pioneering calculations of Asada and Blügel but to determine the magnetic ground states and the corresponding IEC in the $\mathrm{Ni} / \mathrm{Fe} / \mathrm{Co}$ superlattices, so that only the most stable magnetic configurations (Fig. 1) are given here. Nevertheless, for completeness, we report in Table I all the self-consistent solutions with their relative energies obtained in the case of $4 \mathrm{Fe}$ ML's. In that case, we obtained four converged solutions for AF-IEC and three for FM-IEC arrangements between Co and Ni slabs.

Coming back to Fig. 1 which gives the magnetic moments distribution for the most stable configurations of the superlattices under consideration, one can deduce the following main points:

TABLE I. Self-consistent solutions obtained in the case of $\mathrm{Ni}_{5} / \mathrm{Fe}_{4} / \mathrm{Co}_{5}(001)$ superlattices for $\mathrm{AF}$ and $\mathrm{FM}$ arrangements between Ni and Co slabs with their relative energies. $\uparrow(\downarrow)$ stands for "spin-up" and "spin-down" of iron atoms whereas $\Uparrow(\downarrow)$ denotes the spin orientation of $\mathrm{Ni}$ and Co slabs at the left and right of the $\mathrm{Fe}$ spacer, respectively. Two magnetically inequivalent atoms per plane have been considered.

\begin{tabular}{lcc}
\hline \hline & Magnetic configurations & Relative energies (eV) \\
\hline AF-IEC & $\Uparrow \uparrow \uparrow \uparrow \uparrow \uparrow \uparrow \downarrow \downarrow \downarrow$ & 0.82 \\
& $\Uparrow \uparrow \uparrow \downarrow \downarrow \downarrow \downarrow \downarrow \downarrow \downarrow$ & 0.59 \\
& $\Uparrow \uparrow \uparrow \uparrow \uparrow \downarrow \downarrow \downarrow \downarrow \downarrow$ & 0.26 \\
& $\Uparrow \uparrow \uparrow \downarrow \downarrow \uparrow \uparrow \downarrow \downarrow \downarrow$ & 0.0 \\
FM-IEC & $\Uparrow \uparrow \uparrow \downarrow \downarrow \downarrow \downarrow \uparrow \uparrow \Uparrow$ & 0.16 \\
& $\Uparrow \uparrow \uparrow \downarrow \downarrow \uparrow \uparrow \uparrow \uparrow \uparrow$ & 0.30 \\
& $\Uparrow \uparrow \uparrow \uparrow \uparrow \downarrow \downarrow \uparrow \uparrow \uparrow$ & 0.47 \\
\hline \hline
\end{tabular}

(i) A ferromagnetic coupling between $\mathrm{Fe}$ and $\mathrm{Co}$ and between $\mathrm{Fe}$ and $\mathrm{Ni}$ is generally obtained at the interfaces. We notice that when we start the calculations with an antiparallel alignment of magnetic moments at the $\mathrm{Fe} / \mathrm{Ni}$ or $\mathrm{Fe} / \mathrm{Co}$ interfaces, we observe a spin flip of both $\mathrm{Ni}$ and Co interface atoms in order to reach a ferromagnetic coupling with Fe. It is only for $n=1$, and for an antiferromagnetic coupling between the $\mathrm{Co}$ and $\mathrm{Ni}$ slabs, that the $\mathrm{Fe}$ layer presents an in-plane antiferromagnetic configuration.

(ii) An increase of the Fe magnetic moments, as compared to bulk fcc value, is in general observed. This increase is more pronounced at the interfaces with $\mathrm{Co}$ and Ni. Furthermore the $\mathrm{Fe}$ magnetic moment at the $\mathrm{Fe} / \mathrm{Ni}$ interface is always greater than that one at the $\mathrm{Fe} / \mathrm{Co}$ interface and the difference is sometimes large $\left(0.39 \mu_{B}\right.$ for $\left.n=3\right)$. This behavior is somewhat strange because the Co moments being larger than those of $\mathrm{Ni}$, so one could expect the opposite. In fact, it is directly linked to a more stronger Fe-Co hybridization because the $\mathrm{Fe}-\mathrm{Co}$ bands centers are energetically more closer than for $\mathrm{Fe}-\mathrm{Ni}$ bands. More details will be given in Sec. IV.

(iii) A small decrease of the Co moments at the interfaces with Fe is observed.

(iv) Ferromagnetic arrangements in the Fe spacer are only obtained for $n=1$ and 2 and for the FM coupling between $\mathrm{Ni}$ and Co slabs. However only the ferromagnetic configuration for $n=1$ is stable.

(v) The $c(2 \times 2)$ or in-plane antiferromagnetic configuration for Fe appears only in one single Fe layer. For an odd value of $n$ it is always located at the center of the Fe slab. It is the ground state for $n=5$ and 7 where the most stable solutions are AF arrangement between $\mathrm{Ni}$ and Co slabs. Furthermore, in the case of $n=5$ it appears in both FM and AF configurations. For an even value of $n$, the $c(2 \times 2)$ arrangement appears only for $n=6$ and only for the most stable FM 

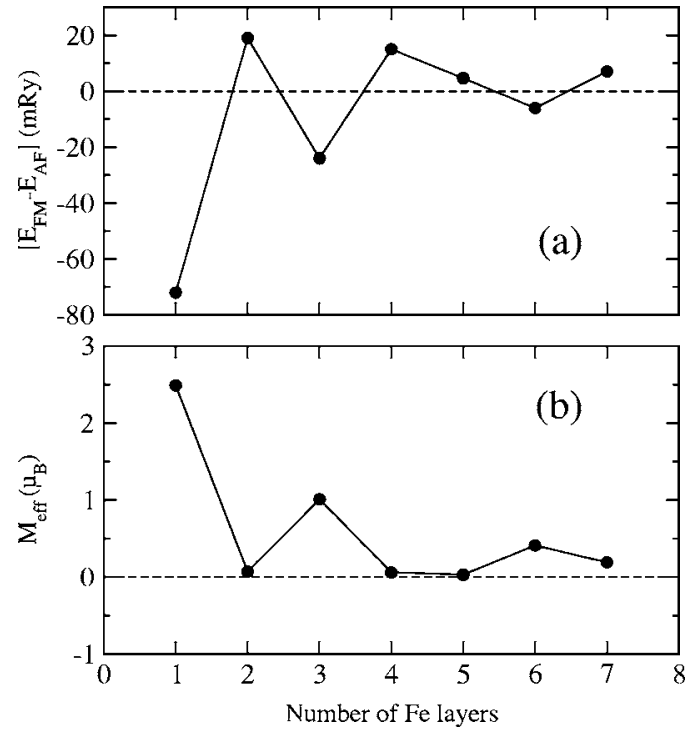

FIG. 2. The energy difference $E_{\mathrm{FM}}-E_{\mathrm{AF}}$ versus the number $n$ of Fe layers for $\mathrm{Ni}_{5} / \mathrm{Fe}_{n} / \mathrm{Co}_{5}(100)$ superlattice. $E_{\mathrm{FM}}$ and $E_{\mathrm{AF}}$ are, respectively, the energy of a FM and AF interlayer exchange coupling between Co and Ni through the Fe spacer (a). Mean magnetic moment $\left(M_{\mathrm{eff}}\right)$ of the Fe film as a function of the number $n$ for the most stable configuration (b).

solution. It is located in the Fe layer at the third nearest neighboring to the Co interface.

(vi) We observe an antiferromagnetically layered configuration inside the Fe films for $n=4,6,7$. It is stable only for $n=4$.

In Fig. 2(a) we have reported the corresponding IEC between $\mathrm{Ni}$ and $\mathrm{Co}$ slabs in terms of their energy difference $E_{\mathrm{FM}}-E_{\mathrm{AF}}$ for $\mathrm{Ni}_{5} / \mathrm{Fe}_{n} / \mathrm{Co}_{5}(100)$ superlattices. The behavior is very similar to that obtained in the case of $\mathrm{Ni}_{5} / \mathrm{Fe}_{n} / \mathrm{Ni}_{5}(100)$ superlattices. ${ }^{23}$ The fcc Fe film mediates a periodic decreasing oscillatory IEC up to $n=4$ where the coupling is of AF-type. From $n=5$, where the coupling between $\mathrm{Co}$ and $\mathrm{Ni}$ slabs is also of AF type, the IEC takes again a periodic oscillatory feature.

We have also reported [Fig. 2(b)] the mean magnetic moment per Fe atom $\left(M_{\text {eff }}\right)$ in terms of the number of Fe layers in the spacer for the most stable configurations. A clear tendency towards antiferromagnetism is observed.

\section{MIXED INTERFACES}

The $\mathrm{Fe} / \mathrm{Co}$ and $\mathrm{Fe} / \mathrm{Ni}$ interfaces are undoubtedly not atomically sharp, and both Co and Ni show a large mutual solubility with $\mathrm{Fe},{ }^{30}$ so that some intermixing at the interfaces is unavoidable. It was previously shown ${ }^{23}$ that some kind of interdiffusion in $\mathrm{Ni} / \mathrm{Fe} / \mathrm{Ni}(100)$ superlattices stabilizes the system. In order to prospect in this direction, we have considered the effect of one layer thick ordered alloy, at both $\mathrm{Fe} / \mathrm{Co}$ and $\mathrm{Fe} / \mathrm{Ni}$ interfaces. We have considered the following superlattices $\mathrm{Ni}_{4} / \mathrm{Fe}_{0.5} \mathrm{Ni}_{0.5} / \mathrm{Fe}_{n-1} / \mathrm{Fe}_{0.5} \mathrm{Co}_{0.5} / \mathrm{Co}_{4}$ for $n=1-7$. These lattices being chemically equivalent to $\mathrm{Ni}_{5} / \mathrm{Fe}_{n} / \mathrm{Co}_{5}$ but with different structural arrangement, allow a direct comparison between their respective total energies.
We remind that different local atomic arrangements exist depending on the number $n$ of Fe layers (even or odd) (Fig. 5 of Ref. 23). If the number $n$ is even (odd number of pure iron layers), two different cases of covering interface planes are distinguished: symmetric and asymmetric arrangements. The symmetric arrangement where $\mathrm{Ni}(\mathrm{Co})$ and $\mathrm{Fe}$ atoms are ordered in identical sublattices of $c(2 \times 2)$ type at both interfaces, it is energetically favored only by $5 \mathrm{mRy}$ than the asymmetric arrangement. In this last one the sublattices at the two interfaces are occupied by opposite types of atoms $[\mathrm{Ni}(\mathrm{Co})$ or $\mathrm{Fe}]$. In the case where $n$ is odd (even number of pure Fe layers) there is no distinction with respect to an occupation of different sublattices with different types of atoms.

Our calculations show that the system with mixed interfaces is not always the most energetically preferred contrary to the symmetric $\mathrm{Ni}_{5} / \mathrm{Fe}_{n} / \mathrm{Ni}_{5}$ system. ${ }^{23}$ Nevertheless, only one ordered $\mathrm{Fe}_{0.5} \mathrm{Ni}_{0.5}\left(\mathrm{Co}_{0.5}\right)$ one layer thick was considered because of the great number of inequivalent atoms. It is obvious that we cannot consider all the possible compositional orderings at the $\mathrm{Ni}(\mathrm{Co})-\mathrm{Fe}$ interfaces essentially because of the great number of stoichiometric $\mathrm{Ni}(\mathrm{Co})-\mathrm{Fe}$ composition that could form at the interfaces. Molecular dynamic simulations and possible relaxation effects, which could clarify the atomic microstructure of the interface, is out of the scope of the TB-LMTO-ASA method. Here also we restrict our discussion only to the most stable magnetic configurations for AF as for FM coupling between Ni and Co slabs. The magnetic map, obtained in this case and reported in Fig. 3 shows the following:

(i) The $\mathrm{Fe}$ polarization in the $\mathrm{Fe}_{0.5} \mathrm{Ni}_{0.5}\left(\mathrm{Co}_{0.5}\right)$ ordered monolayer is always ferromagnetically aligned with $\mathrm{Ni}(\mathrm{Co})$. Moreover, for all configurations considered here (stable or metastable), and for both FM and AF Co-Ni coupling, we have found a strong ferromagnetic Fe-Ni coupling between $\mathrm{Fe}_{0.5} \mathrm{Ni}_{0.5}$ ordered layer and the first pure $\mathrm{Fe}$ monolayer at the nearest neighboring position, whereas for $\mathrm{Fe}-\mathrm{Co}$ this is not truly satisfied for $n=2$ and 3 . Indeed, for $n=2$, where the antiferromagnetic IEC is the ground state the strong $\mathrm{Fe}-\mathrm{Ni}$ interaction align the moment of the single pure Fe layer parallel to the Ni moments. More precisely, for $n=2$, there is an antiferromagnetic coupling between the $\mathrm{Fe}$ moment in the $\mathrm{Fe}_{0.5} \mathrm{Co}_{0.5}$ monolayer and the $\mathrm{Fe}$ moment in the Fe layer in the middle of the spacer. Furthermore, in the case of $n=3$ and with a FM-IEC configuration (metastable), the AF layered ordering in the pure Fe film is correlated with the strong Fe-Ni FM coupling to align antiparallelly the moments of the $\mathrm{Fe}-\mathrm{Co}$ interface and its adjacent $\mathrm{Fe}$ pure monolayer.

(ii) As for the perfect interfaces, an increase of the magnetic moment at the $\mathrm{Fe}$ sites inside the Fe pure spacer is observed, the bigger increase being near the interface with $\mathrm{Ni}$ and $\mathrm{Co}$. This increase is even more pronounced at the $\mathrm{Fe}-\mathrm{Ni}$ interfaces. This result which appears somewhat surprising since Co atoms bear larger magnetic moments, agree well with $\mathrm{Wu}$ et al. ${ }^{14}$ experimental results who observed that the $\mathrm{Fe}$ magnetic moment follows always that of $\mathrm{Ni}$, either in ferromagnetic coupling or in the antiferromagnetic coupling between $\mathrm{Ni}$ and Co slabs. At the alloyed layers, the Fe magnetic moment is considerably augmented, the greatest values being always found at $\mathrm{Fe}_{0.5} \mathrm{Ni}_{0.5}$ layers (Table II). For Ni 


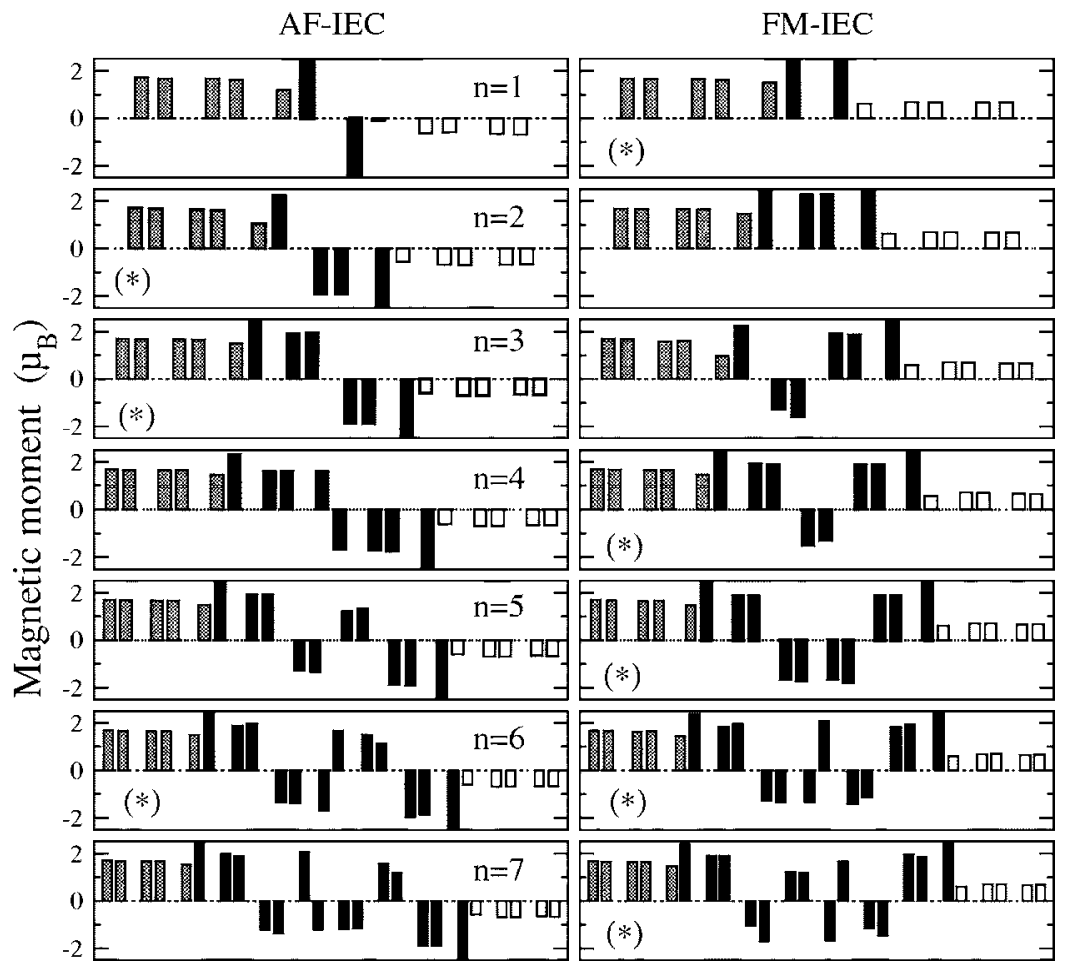

FIG. 3. Magnetic profiles (in $\left.\mu_{B}\right) \quad$ of $\quad \mathrm{Ni}_{4} / \mathrm{Fe}_{0.5} \mathrm{Ni}_{0.5} / \mathrm{Fe}_{n-1}$ $/ \mathrm{Fe}_{0.5} \mathrm{Co}_{0.5} / \mathrm{Co}_{4}(n=1, \ldots, 7)$ superlattices for $\mathrm{AF}$ and $\mathrm{FM}$ alignment between $\mathrm{Co}$ and $\mathrm{Ni}$ slabs (left and right panels, respectively). Dark, gray and open bars represent the values of the magnetic moments of the $\mathrm{Fe}$, $\mathrm{Co}$, and $\mathrm{Ni}$ atoms, respectively. The most stable configuration is indicated by (*); for $n=6$, the two magnetic configurations have the same energy. Two magnetically inequivalent atoms per plane have been considered.

atoms in the $\mathrm{Fe}_{0.5} \mathrm{Ni}_{0.5}$ layer, the magnetic moment decreases marginally from its bulk value $(<6 \%)$. On the contrary, the $\mathrm{Co}$ atoms in the $\mathrm{Fe}_{0.5} \mathrm{Co}_{0.5}$ layer undergo a sizable decrease in their magnetic moments. The decrease is more pronounced on the Co atoms having Fe atoms at the first neighbor sites with antiparallel spin. It is the case for $n=2$ with AF alignment and for $n=3$ with FM coupling between $\mathrm{Ni}$ and $\mathrm{Co}$ slabs, where such Co atoms bear the lowest magnetic moments, 1.05 and $0.98 \mu_{B}$, respectively (Fig. 3). The increase of the interfacial Fe moments which is more important at the $\mathrm{Ni}$ neighbors can be understood through the local density of states (LDOS) of corresponding atoms. Indeed, this is the result of the more or less strong hybridization between the $\mathrm{Fe}$ and $\mathrm{Ni}(\mathrm{Co})$ atoms, in the ordered $\mathrm{Fe}_{0.5} \mathrm{Ni}_{0.5}\left(\mathrm{Co}_{0.5}\right)$ layers. To be more precise we have plotted, in Fig. 4, the LDOS of the interfacial $\mathrm{Fe}$ atoms with $\mathrm{Co}$ [Fig. 4(a)] and $\mathrm{Ni}$ [Fig. 4(b)], for $n=3$ with FM-IEC coupling (Fig. 3), and compared it with the LDOS of fcc-Fe in the bulk phase [Fig. 4(c)]. The spin-up LDOS on the Fe atoms is strongly modified: the Fe-peak above the Fermi level, present in the bulk case, is mainly suppressed when Fe is in contact with $\mathrm{Ni}$. This arises

TABLE II. The Fe, Ni, and Co magnetic moments (in $\mu_{B}$ ) at the ordered alloyed layers for the ground states configurations: (a) at $\mathrm{Fe}_{0.5} \mathrm{Ni}_{0.5}$ layer, (b) at $\mathrm{Fe}_{0.5} \mathrm{Co}_{0.5}$ layer. The bulk values are 1.0, 0.63 , and $1.63 \mu_{B}$ for fcc $\mathrm{Fe}, \mathrm{Ni}$, and $\mathrm{Co}$, respectively.

\begin{tabular}{lllllllll}
\hline \hline & $n$ & 1 & 2 & 3 & 4 & 5 & 6 & 7 \\
\hline (a) & $\mathrm{Ni}$ & 0.63 & 0.57 & 0.59 & 0.57 & 0.59 & 0.60 & 0.60 \\
& $\mathrm{Fe}$ & 2.67 & 2.63 & 2.62 & 2.50 & 2.62 & 2.63 & 2.63 \\
& & & & & & & & \\
(b) & $\mathrm{Fe}$ & 2.48 & 2.26 & 2.42 & 2.38 & 2.37 & 2.43 & 2.37 \\
& $\mathrm{Co}$ & 1.50 & 1.05 & 1.45 & 1.45 & 1.46 & 1.50 & 1.46 \\
\hline \hline
\end{tabular}

because the centers of the $\mathrm{Ni}$ and $\mathrm{Fe} d$-bands are more separated than in the $\mathrm{Fe}-\mathrm{Co}$ case, so that the width of the Fe band is smaller. It is also more peaky because most of Fe neigh-

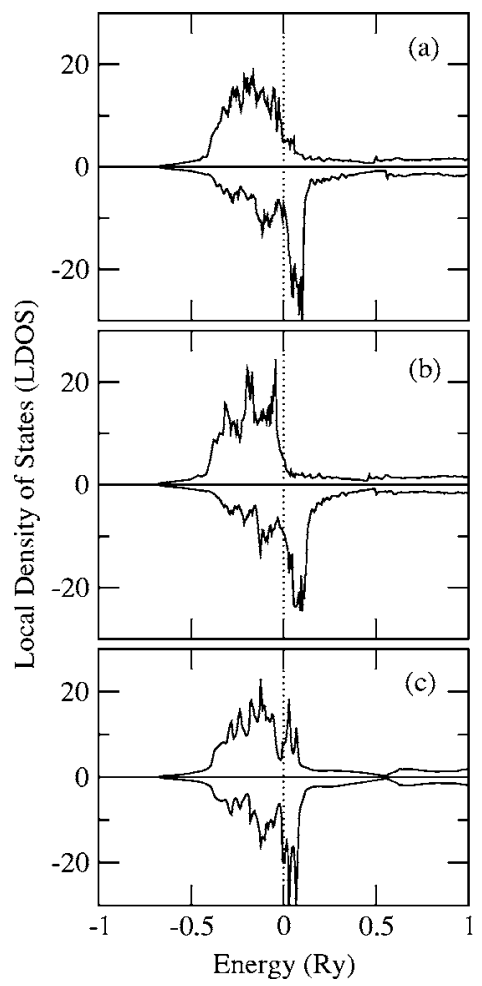

FIG. 4. Local density of states (LDOS) of the interfacial Fe atom in the intermixed layer $\mathrm{Fe}_{0.5} \mathrm{Co}_{0.5}$ (a) and $\mathrm{Fe}_{0.5} \mathrm{Ni}_{0.5}$ (b) in $\mathrm{Ni}_{4} / \mathrm{Fe}_{0.5} \mathrm{Ni}_{0.5} / \mathrm{Fe}_{2} / \mathrm{Fe}_{0.5} \mathrm{Co}_{0.5} / \mathrm{Co}_{4}$ superlattice with $\mathrm{FM}$ coupling between $\mathrm{Ni}$ and Co slabs, compared to the DOS of fcc bulk Fe (c). The vertical dotted line is the Fermi energy. 
bors are $\mathrm{Ni}$ atoms whose $d$-band centers are more distant in energy than those of Co atoms. Normally a $d$-band which is more than half-filled increases its number of electrons when the $d$ - $d$ overlap shrinks. This explains why the number of $d$-electrons increases when $\mathrm{Fe}$ goes from Co to Ni neighborhood. Similar reasoning holds for the spin-down band but now the $d$-bands are less than half-filled. Therefore the number of $d$-electrons are decreasing when the $\mathrm{Fe}$ atom goes from Co to Ni neighborhood. To sum up, it is clear that the magnetic moment on $\mathrm{Fe}$ atoms are higher at the $\mathrm{Ni}$ side.

(iii) A $c(2 \times 2)$ configuration for $\mathrm{Fe}$, one layer thick, is present for $n>3$. This configuration appears in the central layer for $n=4,6$ (corresponding to an odd number of pure $\mathrm{Fe}$ layers) and for $n=7$ (even number of pure Fe layers) it appears in one of the two central Fe layers: for the most stable solution, it is located in the third neighboring layer of the Fe-Ni interface, and for the metastable configuration it is shifted one layer towards the Co side. Contrary to the case of abrupt interfaces where the antiferromagnetic layered coupling inside the Fe spacer seems to be preferred, this type of configuration is destroyed by the presence of the $c(2 \times 2)$ configuration.

(iv) Ferromagnetic configuration for Fe films and for the whole superlattice is only present up to $n=2$. Moreover only the configuration for $n=1$ is stable.

(v) Ferromagnetic coupling between the two central $\mathrm{Fe}$ monolayers inside Fe film and far from interfaces, already found in the $\mathrm{Ni} / \mathrm{Fe}_{4} / \mathrm{Co}$ system (Fig. 1) as a stable solution, is also present for $n=5$ (corresponding to 4 pure Fe layers) but this magnetic configuration is unstable.

In Fig. 5(a), we report the IEC for the $\mathrm{Ni}_{4} / \mathrm{Fe}_{0.5} \mathrm{Ni}_{0.5} / \mathrm{Fe}_{n-1} / \mathrm{Fe}_{0.5} \mathrm{Co}_{0.5} / \mathrm{Co}_{4}$ superlattices. From this figure we can notice a drastic effect resulting from the interdiffusion. Indeed, except for $n=1$ and 2, it is clear from Figs. 2(a) and 5(a) that all configurations with FM coupling in the abrupt case, become AF coupled in the case of intermixing interfaces. We notice however an almost degenerated solution for $n=6$. The mean magnetic moment per Fe atom [Fig. 5(b)] shows a steep decrease from $2.5 \mu_{B}$ for $n=1$, to zero for $n=3$ and then tends to stabilize around $0.55 \mu_{B}$ for greater thickness. The strong sensitivity of IEC to the quality of interfaces is by no means a special property of $\mathrm{Ni} / \mathrm{Fe} / \mathrm{Co}$ system and has been already reported by a number of authors. We can cite the works of Freyss et al. ${ }^{24}$ Turek et al. ${ }^{31}$ on the $\mathrm{Fe} / \mathrm{Cr}(001)$ system which is the most frequently studied in this context. Also Uzdin and Demangeat ${ }^{32}$ have shown that this short-wavelengh IEC in $\mathrm{Fe} / \mathrm{Cr}$ multilayers can be manipulated via interface alloying.

\section{CONCLUSION}

We have discussed the magnetic map and the interlayer exchange coupling between $\mathrm{Co}$ and $\mathrm{Ni}$ through the $\mathrm{Fe}$ spacer in $\mathrm{Ni} / \mathrm{Fe} / \mathrm{Co}(100)$ superlattices. These calculations were performed in order to shed some light on recent PEEM and XMCD experiments displaying a complex magnetic behavior of the $\mathrm{Fe}$ spacer. This work is also a part of a more

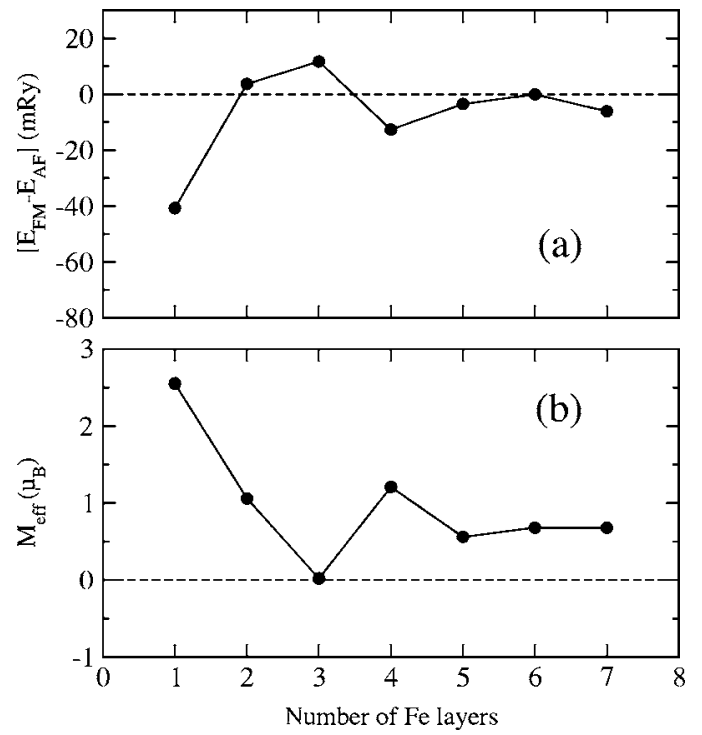

FIG. 5. The energy differences $E_{\mathrm{FM}}-E_{\mathrm{AF}}$ versus the number $n$ of Fe layers for $\mathrm{Ni}_{4} / \mathrm{Fe}_{0.5} \mathrm{Ni}_{0.5} / \mathrm{Fe}_{n-1} / \mathrm{Fe}_{0.5} \mathrm{Co}_{0.5} / \mathrm{Co}_{4}(100)$ superlattices. $E_{\mathrm{FM}}$ and $E_{\mathrm{AF}}$ are, respectively, the energy of a FM and $\mathrm{AF}$ interlayer exchange coupling between $\mathrm{Co}$ and $\mathrm{Ni}$ through the $\mathrm{Fe}$ spacer (a). Mean magnetic moment $\left(M_{\text {eff }}\right)$ of the Fe film as a function of the number $n$ for the most stable configuration (b).

general study of low dimensional superlattices based on $\mathrm{Fe}^{33}$

Our results show that the Fe magnetic moments are enhanced at the interfaces, the greatest increase being always near the $\mathrm{Ni}$ atoms in agreement with $\mathrm{Wu}$ et al. ${ }^{14}$ experiments. Furthermore, we found that, in both perfect and mixed interfaces, the $\mathrm{Fe}-\mathrm{Ni}(\mathrm{Co})$ coupling is always (except for $n=1$ ) ferromagnetic whereas inside the $\mathrm{Fe}$ spacer we observe mainly an antiferromagnetic arrangement. These antiferromagnetic arrangements can be either layered antiferromagnetic between the Fe planes or in-plane $c(2 \times 2)$ one monolayer thick. The IEC behavior depends on the types of interfaces considered: abrupt or alloyed. It is also very different from that obtained in the symmetric $\mathrm{Ni}_{5} / \mathrm{Fe}_{n} / \mathrm{Ni}_{5}$ system. It is clear that the alloying at the interface has a dramatic effect not only on the IEC but also on the average magnetic moment (per Fe atom). Furthermore, the intermixing at $\mathrm{Fe} / \mathrm{Ni}(\mathrm{Co})$ interfaces strongly modifies the IEC behavior. Indeed, for the abrupt interfaces, ferromagnetic coupling between $\mathrm{Ni}$ and $\mathrm{Co}$ is obtained for $n=1,3,6$ and antiferromagnetic coupling elsewhere whereas for the alloyed interfaces the ground state configurations were found ferromagnetically coupled for $n=4,5,7$. Additional structural studies with molecular dynamic simulations are needed to verify the growth modes and clarify the complexity of the coupling behavior between $\mathrm{Ni}$ and Co films across fcc Fe spacer.

\section{ACKNOWLEDGMENTS}

This work was supported by the Algerian Project No. D1501/51/05 and by NATO-Grant No. PST.CLG.979708. We would like to thank Dr. M. A. Khan for fruitful discussions. 
${ }^{1}$ T. Veres, M. Cai, R. W. Cochrane, M. Rouabhi, S. Roorda, and P. Desjardins, Thin Solid Films 382, 172 (2001).

${ }^{2}$ G. B. Fratucello, A. M. Prandini, P. Luches, S. D'Addato, A. J. Patchett, and S. Nannarone, J. Magn. Magn. Mater. 210, 349 (2000).

${ }^{3}$ A. S. Edelstein, C. Kim, S. B. Qadri, K. H. Kim, V. Browning, H. Y. Yu, B. Maruyama, and P. K. E. Verett, Solid State Commun. 76, 1379 (1990).

${ }^{4}$ W. Kuch and S. S. Parkin, J. Magn. Magn. Mater. 184, 127 (1999).

${ }^{5}$ G. B. Fratucello and A. M. Prandini, J. Electron Spectrosc. Relat. Phenom. 76, 665 (1995).

${ }^{6}$ B. R. Acharya, S. N. Piramanayagam, A. K. Nigam, S. N. Shringi, Shiva Prasa, N. Venkataramani, Girich Chandra, and R. Krishman, J. Magn. Magn. Mater. 140-144, 555 (1995).

${ }^{7}$ Ernesto J. Escorcia-Aparicio, R. K. Kawakami, and Z. Q. Qiu, Phys. Rev. B 54, 4155 (1996).

${ }^{8}$ A. Dallmeyer, K. Maiti, O. Rader, L. Pasquali, C. Carbone, and W. Eberhardt, Phys. Rev. B 63, 104413 (2001).

${ }^{9}$ Y. Kakehashi, O. Jepsen, and N. Kimura, Phys. Rev. B 65 , 134418 (2002).

${ }^{10}$ M. Marsman and J. Hafner, Phys. Rev. B 66, 224409 (2002).

${ }^{11}$ D. Spisák and J. Hafner, Phys. Rev. B 67, 134434 (2003).

${ }^{12}$ Kenta Amemiya, Daiju Matsumura, Hitoshi Abe, Soichiro Kitagawa, Toshiaki Ohta, and T. Yokoyama, Phys. Rev. B 70, 195405 (2004).

${ }^{13}$ W. Kuch, J. Gilles, F. Offi, S. S. Kang, S. Imada, S. Suga, and J. Kirschner, J. Electron Spectrosc. Relat. Phenom. 109, 249 (2000).

${ }^{14}$ Y. Z. Wu, C. Won, A. Scholl, A. Doran, F. Toyoma, X. F. Jin, N. V. Smith, and Z. Q. Qiu, Phys. Rev. B 65, 214417 (2002).

${ }^{15}$ C. Won, Y. Z. Wu, A. Scholl, A. Doran, N. Kurahashi, H. W. Zhao, and Z. Q. Qiu, Phys. Rev. Lett. 91, 147202 (2003).
${ }^{16}$ C. Won, Y. Z. Wu, H. W. Zhao, A. Scholl, A. Doran, and Z. Q. Qiu, Phys. Rev. B 68, 052404 (2003).

${ }^{17}$ A. Mokrani, O. Elmouhssine, G. Moraitis, and C. Demangeat, J. Magn. Magn. Mater. 198-199, 561 (1999).

${ }^{18}$ D. Spisák and J. Hafner, Phys. Rev. B 62, 9575 (2000).

${ }^{19}$ C. S. Wang, B. M. Klein, and H. Krakauer, Phys. Rev. Lett. 54, 1852 (1985).

${ }^{20}$ G. H. O. Daalderop, P. J. Kelly, and F. J. A. den Broeder, Phys. Rev. Lett. 68, 682 (1992).

${ }^{21}$ Y. Nagai, M. Senda, and T. Toshima, J. Appl. Phys. 63, 1136 (1988).

${ }^{22}$ C. Prados, D. V. Dimitrov, G. C. Hadjipanayis, and A. Hermando, J. Magn. Magn. Mater. 177-181, 1293 (1998).

${ }^{23}$ A. Hadj-Larbi, S. Bouarab, and C. Demangeat, Phys. Rev. B 66, 144428 (2002).

${ }^{24}$ M. Freyss, D. Stoeffler, and H. Dreyssé, Phys. Rev. B 56, 6047 (1997).

${ }^{25} \mathrm{Ph}$. Mavropoulos, S. Lounis, R. Zeller, and S. Blügel, Appl. Phys. A (in press).

${ }^{26}$ O. K. Andersen and O. Jepsen, Phys. Rev. Lett. 53, 2571 (1984).

${ }^{27}$ O. K. Andersen, Z. Pawlowska, and O. Jepsen, Phys. Rev. B 34, 5253 (1986).

${ }^{28}$ M. Methfessel, C. O. Rodriguez, and O. K. Andersen, Phys. Rev. B 40, R2009 (1989).

${ }^{29}$ T. Asada and S. Blügel, Phys. Rev. Lett. 79, 507 (1997).

${ }^{30}$ W. L. O’Brien and B. P. Tonner, Phys. Rev. B 52, 15332 (1995).

${ }^{31}$ I. Turek, M. Freyss, P. Weinberger, D. Stoeffler, and H. Dreyssé, Phys. Rev. B 63, 024413 (2001).

${ }^{32}$ V. M. Uzdin and C. Demangeat, Phys. Rev. B 66, 092408 (2002).

${ }^{33}$ A. Vega, J. C. Parlebas, and C. Demangeat, in Handbook of Magnetic Materials, edited by K. H. J. Buschow (Elsevier, Amsterdam, 2003), Vol. 15, p. 199. 